\title{
Some new upper bounds for moduli of eigenvalues of iterative matrices
}

\author{
Jun He*, Yanmin Liu, Guangjun Xu \\ School of mathematics, Zunyi Normal College, Zunyi, Guizhou, 563006 China \\ *Corresponding author, e-mail: hejunfan1@163.com
}

Received 9 Feb 2021

Accepted 8 Aug 2021

\begin{abstract}
Based on the matrix splitting $M=P-Q$, some upper bound for the maximum of moduli of eigenvalues of the iteration matrix $P^{-1} Q$ are obtained when $P$ is an strictly diagonally dominant (SDD) matrix or a doubly strictly diagonally dominant (DSDD) matrix. In this paper, some new upper bounds are introduced, which is applicable to a Dashnic-Zusmanovich (DZ) matrix $P$, and proved to be better than those in Huang and Gao [Int $\mathrm{J}$ Comput Math $\mathbf{8 0}$ (2003):799-803] and Li et al [Appl Math Comput 173 (2006):977-984] in certain cases.
\end{abstract}

KEYWORDS: iteration matrix, eigenvalues, spectral radius

MSC2010: 65F50

\section{INTRODUCTION}

Let $M=\left(m_{t s}\right)$ be an $n \times n$ nonsingular complex matrix and consider the following large system of linear equations

$$
M x=b,
$$

which plays an important role in the numerical solution of elliptic partial differential equations $[1,2]$. Based on the matrix splitting $M=P-Q$, some basic iterative methods, such as Jacobi, Gauss-Seidel and successive overrelaxation iterative methods, are introduced to solve the system of linear equations (1) [3]. The matrix splitting $M=P-Q$ leads to the stationary iteration scheme

$$
x_{k+1}=P^{-1} Q x_{k}+c .
$$

The matrix $P^{-1} Q$ in (2) is called the iteration matrix. It is well known that the iteration scheme (2) converges for any initial vector $x_{0}$ if $\rho\left(P^{-1} Q\right)<1$, where $\rho\left(P^{-1} Q\right)$ denotes the spectral radius of the matrix $P^{-1} Q$, namely, maximum of moduli of eigenvalues of $P^{-1} Q$.

Let $\mathbb{C}^{n \times n}$ be the set of all complex matrices, and denote $[n]:=\{1,2, \ldots, n\}$. A matrix $P=\left(p_{t s}\right) \in$ $\mathbb{C}^{n \times n}$ is called an SDD (strictly diagonally dominant) matrix if for each $t \in[n]$,

$$
\left|p_{t t}\right|>r_{t}(P)
$$

where $r_{t}(P)=\sum_{s \neq t}\left|p_{t s}\right|$. A matrix $P=\left(p_{t s}\right) \in \mathbb{C}^{n \times n}$ is called a DSDD (doubly strictly diagonally domi- nant) matrix if for distinct $t, s \in[n]$, the following inequality holds:

$$
\left|p_{t t}\right|\left|p_{s s}\right|>r_{t}(P) r_{s}(P) .
$$

A matrix $P=\left(p_{t s}\right) \in \mathbb{C}^{n \times n}$ is called a DZ (DashnicZusmanovich) matrix if there exists an index $t \in[n]$, for all $s \neq t, s \in[n]$, the following inequality holds:

$$
\left|p_{t t}\right|\left(\left|p_{s s}\right|-r_{s}^{t}(P)\right)>r_{t}(P)\left|p_{s t}\right|,
$$

where $r_{s}^{t}(P)=r_{s}(P)-\left|p_{s t}\right|$. It has been shown in [4] that the above three classes of matrices are nonsingular and have the following inclusions:

$$
\{S D D\} \subseteq\{D S D D\} \subseteq\{D Z\}
$$

One practical application of the eigenvalues of $P^{-1} Q$ is that one can identify the convergence property of the iteration scheme $x_{k+1}=P^{-1} Q x_{k}+c$ by the maximum of moduli of eigenvalues of $P^{-1} Q$, for more details, see [5-8]. In [5,7], the authors established the following result for an SDD matrix $P$.

Theorem $1([5,7])$ Let $P=\left(p_{t s}\right) \in \mathbb{C}^{n \times n}$ be an $S D D$ matrix, $Q=\left(q_{t s}\right) \in \mathbb{C}^{n \times n}$. Then

$$
\left|\lambda\left(P^{-1} Q\right)\right| \leqslant \omega_{1}=\max _{t \in[n]} \frac{\left|q_{t t}\right|+r_{t}(Q)}{\left|p_{t t}\right|-r_{t}(P)} .
$$

If further $P=\left(p_{t s}\right) \in \mathbb{C}^{n \times n}$ is a DSDD matrix, the result in Theorem 1 can be improved as follows. 
Theorem 2 ([8]) Let $P=\left(p_{t s}\right) \in \mathbb{C}^{n \times n}$ be a DSDD matrix, $Q=\left(q_{t s}\right) \in \mathbb{C}^{n \times n}$. Then

$$
\left|\lambda\left(P^{-1} Q\right)\right| \leqslant \omega_{2}=\max _{t, s \in[n], s \neq t} \frac{B+\sqrt{B^{2}-4 A C}}{2 A},
$$

where

$$
\begin{aligned}
& A=\left|p_{t t} p_{s s}\right|-r_{t}(P) r_{s}(P) \\
& B=\left|p_{t t} q_{s s}\right|+\left|q_{t t} p_{s s}\right|+r_{t}(P) r_{s}(Q)+r_{t}(Q) r_{s}(P), \\
& C=\left|q_{t t} q_{s s}\right|-r_{t}(Q) r_{s}(Q)
\end{aligned}
$$

Recently, Li et al [9] presented the following result, for a DSDD matrix $P=\left(p_{t s}\right) \in \mathbb{C}^{n \times n}$.

Theorem 3 ([9]) Let $P=\left(p_{t s}\right) \in \mathbb{C}^{n \times n}$ be a DSDD matrix, $Q=\left(q_{t s}\right) \in \mathbb{C}^{n \times n}$. Then

$$
\left|\lambda\left(P^{-1} Q\right)\right| \leqslant \omega_{3}=\max _{t, s \in[n], s \neq t} \frac{B+\sqrt{B^{2}-4 A C}}{2 A},
$$

where

$$
\begin{aligned}
A & =\left|p_{t t} p_{s s}\right|-r_{t}(P) r_{s}(P), \\
B^{\prime} & =\left|p_{t t} q_{s s}+q_{t t} p_{s s}\right|+r_{t}(P) r_{s}(Q)+r_{t}(Q) r_{s}(P), \\
C^{\prime} & =-\left[\left|q_{t t} q_{s s}\right|+r_{t}(Q) r_{s}(Q)\right] .
\end{aligned}
$$

For an SDD matrix $P=\left(p_{t s}\right) \in \mathbb{C}^{n \times n}$, Theorem 1 can be used to obtain the upper bound for $\left|\lambda\left(P^{-1} Q\right)\right|$. For a DSDD matrix $P$, Theorems 2 and 3 can be used to obtain the upper bounds for $\left|\lambda\left(P^{-1} Q\right)\right|$. In this paper, some new bounds for a DZ matrix $P=\left(p_{t s}\right)$ are introduced.

\section{MAIN RESULTS}

Before presenting our main results, we need the following lemmas.

Lemma 1 ([4]) If $P=\left(p_{t s}\right) \in \mathbb{C}^{n \times n}$ is a DZ matrix, then $P$ is nonsingular.

Lemma 2 ([4]) All eigenvalues of a matrix $P=$ $\left(p_{t s}\right) \in \mathbb{C}^{n \times n}, n \geqslant 2$, belong to the set

$$
\Theta(P)=\bigcap_{t \in[n]} \bigcup_{s \in[n], t \neq s} \Theta_{t s}(P),
$$

where

$\Theta_{t s}(P)=\left\{\lambda \in \mathbb{C}:\left|\lambda-p_{t t}\right|\left(\left|\lambda-p_{s s}\right|-r_{s}^{t}(P)\right)\right\} \leqslant r_{t}(P)\left|p_{s t}\right|$.

Our first main result for a DZ matrix $P$ is stated as follows.
Theorem 4 Let $P=\left(p_{t s}\right) \in \mathbb{C}^{n \times n}$ be a DZ matrix, $Q=$ $\left(q_{t s}\right) \in \mathbb{C}^{n \times n}$. Then

$$
\left|\lambda\left(P^{-1} Q\right)\right| \leqslant \omega_{4}=\min _{t \in[n]} \max _{s \in[n], s \neq t} \frac{H+\sqrt{H^{2}-4 G K}}{2 G},
$$

where

$$
\begin{gathered}
G=\left|p_{t t}\right|\left(\left|p_{s s}\right|-r_{s}^{t}(P)\right)-r_{t}(P)\left|p_{s t}\right|, \\
H=\left|p_{t t}\right|\left(\left|q_{s s}\right|+r_{s}^{t}(Q)\right)+\left|p_{s s}\right|\left|q_{t t}\right| \\
\quad+r_{t}(P)\left|q_{s t}\right|+r_{t}(Q)\left|p_{s t}\right|, \\
K=\left|q_{t t}\right|\left(\left|q_{s s}\right|+r_{s}^{t}(Q)\right)-r_{t}(Q)\left|q_{s t}\right| .
\end{gathered}
$$

Proof: Since $P=\left(p_{t s}\right) \in \mathbb{C}^{n \times n}$ is a DZ matrix, by Lemma $1, P=\left(p_{t s}\right)$ is nonsingular. Assume $\lambda \in$ $\sigma\left(P^{-1} Q\right)\left(\sigma\left(P^{-1} Q\right)\right.$ is the set of all the eigenvalues of $\left.P^{-1} Q\right)$, then

$$
\operatorname{det}\left(\lambda I-P^{-1} Q\right)=0,
$$

which means

$$
\operatorname{det}(\lambda P-Q)=0 \text {. }
$$

By Lemma 2, for the index $t \in[n]$, there is an index $s \in[n], s \neq t$, if

$$
\begin{aligned}
\left|\lambda p_{t t}-q_{t t}\right|\left(\left|\lambda p_{s s}-q_{s s}\right|-r_{s}^{t}(\lambda P-Q)\right) & \\
& >r_{t}(\lambda P-Q)\left|\lambda p_{s t}-q_{s t}\right|,
\end{aligned}
$$

then $\lambda \notin \sigma\left(P^{-1} Q\right)$. Therefore, if

$$
\begin{aligned}
\left(|\lambda|\left|p_{t t}\right|\right. & \left.-\left|q_{t t}\right|\right)\left(|\lambda|\left|p_{s s}\right|-\left|q_{s s}\right|\right) \\
- & \left(|\lambda|\left|p_{t t}\right|+\left|q_{t t}\right|\right)\left(|\lambda| r_{s}^{t}(P)+r_{s}^{t}(Q)\right) \\
& >\left(|\lambda| r_{t}(P)+r_{t}(Q)\right)\left(|\lambda|\left|p_{s t}\right|+\left|q_{s t}\right|\right),
\end{aligned}
$$

then, $\lambda \notin \sigma\left(P^{-1} Q\right)$. Therefore, if $\lambda \in \sigma\left(P^{-1} Q\right)$, we have

$$
\begin{aligned}
\left(|\lambda|\left|p_{t t}\right|-\left|q_{t t}\right|\right)\left(|\lambda|\left|p_{s s}\right|-\left|q_{s s}\right|\right) & \\
- & \left(|\lambda|\left|p_{t t}\right|+\left|q_{t t}\right|\right)\left(|\lambda| r_{s}^{t}(P)+r_{s}^{t}(Q)\right) \\
& \leqslant\left(|\lambda| r_{t}(P)+r_{s}(Q)\right)\left(|\lambda|\left|p_{s t}\right|+\left|q_{s t}\right|\right),
\end{aligned}
$$

that is,

$$
G|\lambda|^{2}-H|\lambda|+K \leqslant 0,
$$

where

$$
\begin{gathered}
G=\left|p_{t t}\right|\left(\left|p_{s s}\right|-r_{s}^{t}(P)\right)-r_{t}(P)\left|p_{s t}\right|, \\
H=\left|p_{t t}\right|\left(\left|q_{s s}\right|+r_{s}^{t}(Q)\right)+\left|p_{s s}\right|\left|q_{t t}\right| \\
\quad+r_{t}(P)\left|q_{s t}\right|+r_{t}(Q)\left|p_{s t}\right|, \\
K=\left|q_{t t}\right|\left(\left|q_{s s}\right|+r_{s}^{t}(Q)\right)-r_{t}(Q)\left|q_{s t}\right| .
\end{gathered}
$$


Since $P=\left(p_{t s}\right) \in \mathbb{C}^{n \times n}$ is a DZ matrix, then $\left|p_{t t}\right|\left(\left|p_{s s}\right|-r_{s}^{t}(P)\right)-r_{t}(P)\left|p_{s t}\right|>0$, then for the index $t \in[n]$, there is an index $s \in[n], s \neq t$,

$$
\left|\lambda\left(P^{-1} Q\right)\right| \leqslant \max _{s \in[n], s \neq t} \frac{H+\sqrt{H^{2}-4 G K}}{2 G},
$$

By the arbitrariness of $t$, we have

$$
\left|\lambda\left(P^{-1} Q\right)\right| \leqslant \min _{t \in[n]} \max _{s \in[n], s \neq t} \frac{H+\sqrt{H^{2}-4 G K}}{2 G} .
$$

Remark 1 In Theorem 4 of [9], when $P=\left(p_{t s}\right) \in$ $\mathbb{C}^{n \times n}$ is a DSDD matrix, $Q=\left(q_{t s}\right) \in \mathbb{C}^{n \times n}$, the authors obtained the following result:

$$
\left|\lambda\left(P^{-1} Q\right)\right| \leqslant \max _{t, s \in[n], s \neq t}\left\{\min \left\{\tau_{1}, \tau_{2}\right\}\right\},
$$

where $\tau_{1}=\frac{B+\sqrt{B^{2}-4 A C}}{2 A}, \tau_{2}=\frac{E+\sqrt{E^{2}-4 D F}}{2 D}$, and

$$
\begin{aligned}
& D=\left|p_{t t} p_{s s}\right|+r_{t}(P) r_{s}(P), \\
& E=\left|p_{t t} q_{s s}+q_{t t} p_{s s}\right|-r_{t}(P) r_{s}(Q)-r_{t}(Q) r_{s}(P), \\
& F=\left|q_{t t} q_{s s}\right|+r_{t}(Q) r_{s}(Q) .
\end{aligned}
$$

If $\Delta=E^{2}-4 D F<0$, the authors take $\frac{E+\sqrt{E^{2}-4 D F}}{2 D}=$ $\infty$. There are some errors in the calculation process of Theorem 4 in [9]. First, from inequality (7) in the proof of Theorem 4 in [9], we have

$$
\begin{aligned}
{\left[\left|p_{t t} p_{s s}\right|+r_{t}(P) r_{s}(P)\right]|\lambda|^{2}-\left[\left|p_{t t} q_{s s}\right|\right.} & \\
& \left.+p_{s s} q_{t t} \mid-r_{t}(P) r_{s}(Q)-r_{s}(P) r_{t}(Q)\right]|\lambda| \\
& +\left[q_{t t} q_{s s}+r_{t}(Q) r_{s}(Q)\right] \geqslant 0,
\end{aligned}
$$

Obviously, by the above inequality, $E$ should be defined as follows:

$$
E=\left|p_{t t} q_{s s}\right|+\left|q_{t t} p_{s s}\right|-r_{t}(P) r_{s}(Q)-r_{t}(Q) r_{s}(P) .
$$

Second, from inequality (7) in the proof of Theorem 4 in [9], we have

$$
D|\lambda|^{2}-E|\lambda|+F \geqslant 0,
$$

if $\Delta \geqslant 0$, then

$$
|\lambda| \leqslant \frac{E-\sqrt{E^{2}-4 D F}}{2 D} \text {, or }|\lambda| \geqslant \frac{E+\sqrt{E^{2}-4 D F}}{2 D},
$$

which means, the result of Theorem 4 in [9] is incorrect.
Another upper bound for moduli of eigenvalues $\left|\lambda\left(P^{-1} Q\right)\right|$ is incorporated in the following theorem.

Theorem 5 Let $P=\left(p_{t s}\right) \in \mathbb{C}^{n \times n}$ be a DZ matrix, $Q=$ $\left(q_{t s}\right) \in \mathbb{C}^{n \times n}$. Then

$$
\left|\lambda\left(P^{-1} Q\right)\right| \leqslant \omega_{5}=\min _{t \in[n]} \max _{s \in[n], s \neq t} \frac{H^{\prime}+\sqrt{H^{\prime 2}-4 G K^{\prime}}}{2 G},
$$

where

$$
\begin{gathered}
G=\left|p_{t t}\right|\left(\left|p_{s s}\right|-r_{s}^{t}(P)\right)-r_{t}(P)\left|p_{s t}\right|, \\
\begin{aligned}
H^{\prime}= & \left|p_{t t} q_{s s}+p_{s s} q_{t t}\right|+\left|p_{t t}\right| r_{s}^{t}(Q) \\
& \quad+\left|q_{t t}\right| r_{s}^{t}(P)+r_{t}(P)\left|q_{s t}\right|+r_{t}(Q)\left|p_{s t}\right|, \\
K^{\prime}=- & \left|q_{t t}\right|\left(\left|q_{s s}\right|+r_{s}^{t}(Q)\right)-r_{t}(Q)\left|q_{s t}\right| .
\end{aligned}
\end{gathered}
$$

Proof: Since $P=\left(p_{t s}\right) \in \mathbb{C}^{n \times n}$ is a DZ matrix, by Lemma $1, p=\left(p_{t s}\right)$ is nonsingular. Assume $\lambda \in$ $\sigma\left(P^{-1} Q\right)$. Then

$$
\operatorname{det}\left(\lambda I-P^{-1} Q\right)=0,
$$

which implies

$$
\operatorname{det}(\lambda P-Q)=0 .
$$

By Lemma 2, for the index $t \in[n]$, there is an index $s \in[n], s \neq t$, if

$$
\begin{aligned}
\left|\lambda p_{t t}-q_{t t}\right|\left(\left|\lambda p_{s s}-q_{s s}\right|-r_{s}^{t}(\lambda P-Q)\right) & \\
& >r_{t}(\lambda P-Q)\left|\lambda p_{s t}-q_{s t}\right|
\end{aligned}
$$

then $\lambda \notin \sigma\left(P^{-1} Q\right)$. Therefore, if

$$
\begin{aligned}
\left(|\lambda|^{2}\left|p_{t t} p_{s s}\right|-\left|p_{t t} q_{s s}+p_{s s} q_{t t}\right||\lambda|-\left|q_{t t} q_{s s}\right|\right) & \\
- & \left(|\lambda|\left|p_{t t}\right|+\left|q_{t t}\right|\right)\left(|\lambda| r_{s}^{t}(P)+r_{s}^{t}(Q)\right) \\
& >\left(|\lambda| r_{t}(P)+r_{t}(Q)\right)\left(|\lambda|\left|p_{s t}\right|+\left|q_{s t}\right|\right)
\end{aligned}
$$

then $\lambda \notin \sigma\left(P^{-1} Q\right)$. Therefore, if $\lambda \in \sigma\left(P^{-1} Q\right)$, we have

$$
\begin{aligned}
\left(|\lambda|^{2}\left|p_{t t} p_{s s}\right|-\left|p_{t t} q_{s s}+p_{s s} q_{t t}\right||\lambda|-\left|q_{t t} q_{s s}\right|\right) & \\
- & \left(|\lambda|\left|p_{t t}\right|+\left|q_{t t}\right|\right)\left(|\lambda| r_{s}^{t}(P)+r_{s}^{t}(Q)\right) \\
& \leqslant\left(|\lambda| r_{t}(P)+r_{t}(Q)\right)\left(|\lambda|\left|p_{s t}\right|+\left|q_{s t}\right|\right)
\end{aligned}
$$

that is,

$$
G|\lambda|^{2}-H^{\prime}|\lambda|+K^{\prime} \leqslant 0,
$$

where

$$
\begin{aligned}
G= & \left|p_{t t}\right|\left(\left|p_{s s}\right|-r_{s}^{t}(P)\right)-r_{t}(P)\left|p_{s t}\right| \\
H^{\prime}= & \left|p_{t t} q_{s s}+p_{s s} q_{t t}\right|+\left|p_{t t}\right| r_{s}^{t}(Q) \\
& +\left|q_{t t}\right| r_{s}^{t}(P)+r_{t}(P)\left|q_{s t}\right|+r_{t}(Q)\left|p_{s t}\right|, \\
K^{\prime}=- & \left|q_{t t}\right|\left(\left|q_{s s}\right|+r_{s}^{t}(Q)\right)-r_{t}(Q)\left|q_{s t}\right| .
\end{aligned}
$$


Since $P=\left(p_{t s}\right) \in \mathbb{C}^{n \times n}$ is a DZ matrix, $G=$ $\left|p_{t t}\right|\left(\left|p_{s s}\right|-r_{s}^{t}(P)\right)-r_{t}(P)\left|p_{s t}\right|>0$, and $K^{\prime} \leqslant 0$. Note that for the index $t \in[n]$, there is an index $t \neq s$, $t \in[n]$,

$$
\left|\lambda\left(P^{-1} Q\right)\right| \leqslant \max _{s \in[n], s \neq t} \frac{H^{\prime}+\sqrt{H^{\prime 2}-4 G K^{\prime}}}{2 G},
$$

By the arbitrariness of $t$, we have

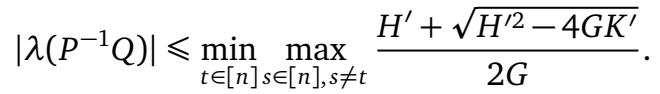

In [9], Li et al presented $\omega_{2} \leqslant \omega_{1}$ when $P=$ $\operatorname{diag}(M)$. In the following, we give some comparison theorems. First, we give the relationships between $\omega_{2}$ and $\omega_{1}$ without the condition $P=$ $\operatorname{diag}(M)$.

Theorem 6 Let $P=\left(p_{t s}\right) \in \mathbb{C}^{n \times n}$ be an SDD matrix, $Q=\left(q_{t s}\right) \in \mathbb{C}^{n \times n}$. Then

$$
\omega_{2} \leqslant \omega_{1} .
$$

Proof: If $|\lambda| \leqslant \omega_{2}$, from the proof of Theorem 3 in [9], there are distinct indices $t, s \in[n]$, such that

$$
\begin{gathered}
\left(\left|p_{t t}\right||\lambda|-\left|q_{t t}\right|\right)\left(\left|p_{s s}\right||\lambda|-\left|q_{s s}\right|\right) \\
\leqslant\left(|\lambda| r_{t}(P)+r_{t}(Q)\right)\left(|\lambda| r_{s}(P)+r_{s}(Q)\right) . \\
\text { If }\left(|\lambda| r_{t}(P)+r_{t}(Q)\right)\left(|\lambda| r_{s}(P)+r_{s}(Q)\right)=0 \text {, then } \\
\left.\quad\left(\left|p_{t t}\right||\lambda|-\left|q_{t t}\right|\right)\left(\left|p_{s s}\right||\lambda|-\left|q_{s s}\right|\right)\right) \leqslant 0,
\end{gathered}
$$

which implies that, one factor in the left side of above inequality is negative. Without loss of generality, we assume $\left|p_{t t}\right||\lambda|-\left|q_{t t}\right| \leqslant 0 \leqslant|\lambda| r_{t}(P)+r_{t}(Q)$, then

$$
|\lambda| \leqslant \omega_{1}=\max _{t \in[n]} \frac{\left|q_{t t}\right|+r_{t}(Q)}{\left|p_{t t}\right|-r_{t}(P)} .
$$

If $\left(|\lambda| r_{t}(P)+r_{t}(Q)\right)\left(|\lambda| r_{s}(P)+r_{s}(Q)\right)>0$, then

$$
\frac{\left|p_{t t}\right||\lambda|-\left|q_{t t}\right|}{|\lambda| r_{t}(P)+r_{t}(Q)} \frac{\left|p_{s s}\right||\lambda|-\left|q_{s s}\right|}{|\lambda| r_{s}(P)+r_{s}(Q)} \leqslant 1 .
$$

Thus,

or

$$
\frac{\left|p_{t t}\right||\lambda|-\left|q_{t t}\right|}{|\lambda| r_{t}(P)+r_{t}(Q)} \leqslant 1
$$

$$
\frac{\left|p_{s s}\right||\lambda|-\left|q_{s s}\right|}{|\lambda| r_{s}(P)+r_{s}(Q)} \leqslant 1
$$

Therefore,

$$
|\lambda| \leqslant \omega_{1}=\max _{t \in[n]} \frac{\left|q_{t t}\right|+r_{t}(Q)}{\left|p_{t t}\right|-r_{t}(P)} .
$$

Second, the relationships among $\omega_{2}, \omega_{3}, \omega_{4}$ and $\omega_{5}$ are also examined.
Theorem 7 Let $P=\left(p_{t s}\right) \in \mathbb{C}^{n \times n}$ be a DSDD matrix, $Q=\left(q_{t s}\right) \in \mathbb{C}^{n \times n}$. Assume that $\left|p_{t t}\right||\lambda|+\left|q_{t t}\right| \leqslant$ $|\lambda| r_{t}(P)+r_{t}(Q)$ for all $t \in[n], \lambda \in \sigma\left(P^{-1} Q\right)$. Then

$$
\omega_{5} \leqslant \omega_{3}, \quad \omega_{4} \leqslant \omega_{2} .
$$

Proof: If $|\lambda| \leqslant \omega_{4}$, and there is an index $t \in[n]$ with $\left|p_{t t}\right||\lambda|+\left|q_{t t}\right| \leqslant|\lambda| r_{t}(P)+r_{t}(Q)$. From the proof of Theorem 4 , for the index $t \in[n]$, there is an index $s \in[n], s \neq t$, we have

$$
\begin{aligned}
& \left(|\lambda|\left|p_{t t}\right|-\left|q_{t t}\right|\right)\left(|\lambda|\left|p_{s s}\right|-\left|q_{s s}\right|\right) \\
& -\left(|\lambda|\left|p_{t t}\right|+\left|q_{t t}\right|\right)\left(|\lambda| r_{s}^{t}(P)+r_{s}^{t}(Q)\right) \\
& \quad \leqslant\left(|\lambda| r_{t}(P)+r_{t}(Q)\right)\left(|\lambda|\left|p_{s t}\right|+\left|q_{s t}\right|\right)
\end{aligned}
$$

Then

$$
\begin{gathered}
\left(|\lambda|\left|p_{t t}\right|-\left|q_{t t}\right|\right)\left(|\lambda|\left|p_{s s}\right|-\left|q_{s s}\right|\right) \\
\leqslant\left(|\lambda| r_{t}(P)+r_{t}(Q)\right)\left(|\lambda|\left|p_{s t}\right|+\left|q_{s t}\right|\right) \\
\quad+\left(|\lambda|\left|p_{t t}\right|+\left|q_{t t}\right|\right)\left(|\lambda| r_{s}^{t}(P)+r_{s}^{t}(Q)\right) \\
\leqslant\left(|\lambda| r_{t}(P)+r_{t}(Q)\right)\left(|\lambda|\left|p_{s t}\right|+\left|q_{s t}\right|\right) \\
\quad+\left(|\lambda| r_{t}(P)+r_{t}(Q)\right)\left(|\lambda| r_{s}^{t}(P)+r_{s}^{t}(Q)\right) \\
\leqslant\left(|\lambda| r_{t}(P)+r_{t}(Q)\right)\left(|\lambda| r_{s}(P)+r_{s}(Q)\right) .
\end{gathered}
$$

Therefore, $|\lambda| \leqslant \omega_{2}$.

If $|\lambda| \leqslant \omega_{5}$, and $\left|p_{t t}\right||\lambda|+\left|q_{t t}\right| \leqslant|\lambda| r_{t}(P)+r_{t}(Q)$.

From the proof of Theorem 5 , for the index $t \in[n]$, there is an index $s \in[n], s \neq t$, such that

$$
\begin{aligned}
& \left(|\lambda|^{2}\left|p_{t t} p_{s s}\right|-\left|p_{t t} q_{s s}+p_{s s} q_{t t}\right||\lambda|-\left|q_{t t} q_{s s}\right|\right) \\
& -\left(|\lambda|\left|p_{t t}\right|+\left|q_{t t}\right|\right)\left(|\lambda| r_{s}^{t}(P)+r_{s}^{t}(Q)\right) \\
& \quad \leqslant\left(|\lambda| r_{t}(P)+r_{t}(Q)\right)\left(|\lambda|\left|p_{s t}\right|+\left|q_{s t}\right|\right)
\end{aligned}
$$

Then

$$
\begin{gathered}
|\lambda|^{2}\left|p_{t t} p_{s s}\right|-\left|p_{t t} q_{s s}+p_{s s} q_{t t}\right||\lambda|-\left|q_{t t} q_{s s}\right| \\
\leqslant\left(|\lambda| r_{t}(P)+r_{t}(Q)\right)\left(|\lambda|\left|p_{s t}\right|+\left|q_{s t}\right|\right) \\
+\left(|\lambda|\left|p_{t t}\right|+\left|q_{t t}\right|\right)\left(|\lambda| r_{s}^{t}(P)+r_{s}^{t}(Q)\right) \\
\leqslant\left(|\lambda| r_{t}(P)+r_{t}(Q)\right)\left(|\lambda|\left|p_{s t}\right|+\left|q_{s t}\right|\right) \\
+\left(|\lambda| r_{t}(P)+r_{t}(Q)\right)\left(|\lambda| r_{s}^{t}(P)+r_{s}^{t}(Q)\right) \\
\leqslant\left(|\lambda| r_{t}(P)+r_{t}(Q)\right)\left(|\lambda| r_{s}(P)+r_{s}(Q)\right) .
\end{gathered}
$$

Therefore, $|\lambda| \leqslant \omega_{3}$.

Remark 2 If $P$ is an SDD matrix, by the condition "| $p_{t t}|| \lambda|+| q_{t t}|\leqslant| \lambda \mid r_{t}(P)+r_{t}(Q)$ for all $t \in[n]$ ", we have

$$
|\lambda| \leqslant \frac{r_{t}(Q)-\left|q_{t t}\right|}{\left|p_{t t}\right|-r_{t}(P)} .
$$

If $P$ is a DSDD matrix and not an SDD matrix, if $\left|p_{t t}\right|>r_{t}(P)$ for some $t \in[n]$, by the condition 
“| $p_{t t}|| \lambda|+| q_{t t}|\leqslant| \lambda \mid r_{t}(P)+r_{t}(Q)$ for all $t \in[n]$ ”, we have

$$
|\lambda| \leqslant \frac{r_{t}(Q)-\left|q_{t t}\right|}{\left|p_{t t}\right|-r_{t}(P)}
$$

If $\left|p_{t t}\right| \leqslant r_{t}(P)$, then we can take $\left|p_{t t}\right| \leqslant r_{t}(P)$, $\left|q_{t t}\right| \leqslant r_{t}(Q)$.

Remark 3 The relationship between $\omega_{4}$ and $\omega_{5}$ is not obvious. But we can find that, if

$$
\operatorname{sign}\left(p_{t t} q_{s s}\right) \operatorname{sign}\left(p_{s s} q_{t t}\right)<0
$$

for distinct $t, s \in[n]$, then $\omega_{4} \leqslant \omega_{5}$, where $\operatorname{sign}(x)$ is the sign function.

\section{NUMERICAL EXAMPLES}

In this section, some numerical examples are given to illustrate the efficiency of our proposed upper bounds.

\section{Example 1 Let}

$$
P=\left[\begin{array}{lll}
7 & 2 & 4 \\
1 & 7 & 1 \\
1 & 1 & 9
\end{array}\right], \quad Q=\left[\begin{array}{lll}
1 & 0 & 3 \\
1 & 0 & 2 \\
1 & 2 & 0
\end{array}\right],
$$

Obviously, $P$ is an SDD matrix. By a direct computation, we have

$$
\rho\left(P^{-1} Q\right)=0.2985 \leqslant \min _{t \in[n]} \frac{r_{t}(Q)-\left|q_{t t}\right|}{\left|p_{t t}\right|-r_{t}(P)}=0.4286,
$$

which means that, the matrix splitting in Example 1 satisfies the condition in Theorem 7.

Example 2 Let

$$
P=\left[\begin{array}{ccc}
-7 & 2 & 1 \\
1 & 7 & 1 \\
1 & 1 & 9
\end{array}\right], \quad Q=\left[\begin{array}{lll}
1 & 0 & 3 \\
0 & 2 & 1 \\
1 & 4 & 1
\end{array}\right],
$$

Obviously, $P$ is an SDD matrix. By a direct computation, we have

$$
\rho\left(P^{-1} Q\right)=0.4175>\min _{t \in[n]} \frac{r_{t}(Q)-\left|q_{t t}\right|}{\left|p_{t t}\right|-r_{t}(P)}=-0.2,
$$

which means that, the matrix splitting in Example 2 does not satisfy the condition in Theorem 7.

\section{Example 3 Let}

$$
P=\left[\begin{array}{ccc}
-7 & 2 & 1 \\
1 & 1.5 & 1 \\
1 & 2 & 2.5
\end{array}\right], \quad Q=\left[\begin{array}{ccc}
1 & 0 & 0.1 \\
0 & 1 & 1 \\
0 & 0 & 1
\end{array}\right],
$$

Obviously, $P$ is not a DSDD matrix. Then, Theorems 1-3 can not be applied in Example 3, but $P$ is a DZ matrix. By a direct computation, we have

$$
\rho\left(P^{-1} Q\right)=0.6993 \text {. }
$$

\section{Example 4 Let}

$$
p=\left[\begin{array}{ccc}
-7 & 2 & 6 \\
0 & 8 & 1 \\
1 & 1 & 9
\end{array}\right], \quad Q=\left[\begin{array}{lll}
1 & 0 & 3 \\
0 & 2 & 1 \\
1 & 0 & 1
\end{array}\right],
$$

Obviously, $P$ is not an SDD matrix. Then Theorem 1 can not be applied in Example 4, but $P$ is a DSDD matrix. If $t=1,\left|p_{11}\right| \leqslant r_{1}(P),\left|q_{11}\right| \leqslant r_{1}(Q)$. If $t=$ 2,3 , by a direct computation, we have

$$
\rho\left(P^{-1} Q\right)=0.2437>\min _{t \in[n]} \frac{r_{t}(Q)-\left|q_{t t}\right|}{\left|p_{t t}\right|-r_{t}(P)}=-0.1429,
$$

which means that, the matrix splitting in Example 4 does not satisfy the condition in Theorem 7.

Example 5 Let

$$
p=\left[\begin{array}{ccc}
-7 & 2 & 6 \\
0 & 8 & 1 \\
1 & 1 & 9
\end{array}\right], \quad Q=\left[\begin{array}{lll}
1 & 0 & 3 \\
1 & 0 & 2 \\
1 & 2 & 0
\end{array}\right],
$$

Obviously, $P$ is not an SDD matrix. Then Theorem 1 can not be applied in Example 5, but $P$ is a DSDD matrix. If $t=1,\left|p_{11}\right| \leqslant r_{1}(P),\left|q_{11}\right| \leqslant r_{1}(Q)$. If $t=$ 2,3 , by a direct computation, we have

$$
\rho\left(P^{-1} Q\right)=0.3011 \leqslant \min _{t \in[2,3]} \frac{r_{t}(Q)-\left|q_{t t}\right|}{\left|p_{t t}\right|-r_{t}(P)}=0.4286
$$

which means that, the matrix splitting in Example 5 satisfies the condition in Theorem 7.

Table 1 summarizes for Examples 1-5 the performance of the upper bounds proposed in this paper comparing with these of $[5,7,9]$. From Table 1 , we can find that, if the matrix splitting satisfies the condition in Theorem 7, then, the result in Theorem 5 is always better than the result in Theorem 2, and the result in Theorem 4 is always better than the result in Theorem 3, which are illustrated in Examples 1 and 5. If $P$ is a DZ matrix, the upper bound can only be obtained by Theorems 4 and 5 .

\section{CONCLUSION}

It is well known that $\{\mathrm{SDD}\} \subseteq\{\mathrm{DSDD}\} \subseteq\{\mathrm{DZ}\}$. In this paper, two upper bounds for $\left|\lambda\left(P^{-1} Q\right)\right|$ (or $\left.\rho\left(P^{-1} Q\right)\right)$ are investigated, which is applicable to a DZ matrix $P$. We also compare our results with some existing results under certain conditions. 
Table 1 Numerical comparison between our upper bounds and those of [5, 7, 9].

\begin{tabular}{l|ccccc}
\hline & Example 1 & Example 2 & Example 3 & Example 4 & Example 5 \\
\hline$\rho\left(P^{-1} Q\right)$ & 0.2985 & 0.4087 & 0.6993 & 0.2437 & 0.3011 \\
Theorem 1 & $4(>1)$ & 1 & - & - & - \\
Theorem 2 & $1.0660(>1)$ & 0.9170 & - & 0.7166 & $1.0179(>1)$ \\
Theorem 3 & $1.0660(>1)$ & 0.7794 & $1.1506(>1)$ & 0.5452 & $1.0179(>1)$ \\
Theorem 4 & $0.9784(<1)$ & 1 & $0.8410(<1)$ & 0.46937 & $0.8515(<1)$ \\
Theorem 5 & $0.9784(<1)$ & 0.8089 & & $0.8515(<1)$ \\
\hline
\end{tabular}

Acknowledgements: This work is supported by NSF of China (71461027, 11661084), Innovative talent team in Guizhou Province (Qian Ke He Pingtai Rencai[2016]5619), New academic talents and innovative exploration fostering project(Qian Ke He Pingtai Rencai[2017]5727-21), Guizhou Province Natural Science Foundation in China (Qian Jiao He KY[2020]094), Science and Technology Foundation of Guizhou Province (Qian Ke He Ji Chu ZK[2021]Yi Ban 014).

\section{REFERENCES}

1. Horn R, Johnson C (1985) Matrix Analysis, Cambridge University Press, Cambridge, UK.

2. Berman A, Plemmons R (1994) Nonnegative Matrices in Mathematical Sciences, SIAM, Philadelphia, PA.

3. Varga R (2000) Matrix Iterative Analysis, Springer, Berlin, Heidelberg.
4. Cvetković L (2006) $H$-matrix theory vs. eigenvalue localization. Numer Algorithms 42, 229-245.

5. Wang X (1994) The upper bound of the spectral radius of $M^{-1} N$ and convergence of some iterative methods. Int $J$ Comput Math 53, 203-217.

6. Wang X (1997) Convergence theory for the general GAOR type iterative method and the MSOR iterative method applied to $H$-matrices. Linear Algebra Appl 250, 1-19.

7. Hu J (1986) The upper and lower bounds of the eigenvalues $M^{1} N$. Math Numer Sin 8, 41-46. [in Chinese]

8. Huang T, Gao Z (2003) A new upper bound for moduli of eigenvalues of iterative matrices. Int J Comput Math 80, 799-803.

9. Li H, Huang T, Li H (2006) An improvement on a new upper bound for moduli of eigenvalues of iterative matrices. Appl Math Comput 173, 977-984. 